\title{
Osthole attenuates ovalbumin-induced lung inflammation via the inhibition of IL-33/ST2 signaling in asthmatic mice
}

\author{
QINGQING YANG $^{1 *}$, LINGWEN KONG $^{2 *}$, WEILING HUANG $^{1}$, NABIJAN MOHAMMADTURSUN $^{2}$, \\ XIUMIN $\mathrm{LI}^{3}$, GUIFANG WANG ${ }^{4}$ and LIXIN WANG ${ }^{5}$
}

\begin{abstract}
${ }^{1}$ Department of Respiratory Medicine, Shanghai Municipal Hospital of Traditional Chinese Medicine, Shanghai University of Traditional Chinese Medicine, Shanghai 200071; ${ }^{2}$ Department of Integrative Medicine, Huashan Hospital, Fudan University, Shanghai 200040, P.R. China; ${ }^{3}$ Department of Pediatrics, Jaffe Food Allergy Institute, Icahn School of Medicine at Mount Sinai, New York, NY 10029, USA; ${ }^{4}$ Department of Respiratory Medicine, Huashan Hospital, Fudan University, Shanghai 200040; ${ }^{5}$ Department of Integrated Traditional Chinese and Western Medicine, Shanghai Pulmonary Hospital, Tongji University, Shanghai 200433, P.R. China
\end{abstract}

Received November 20, 2018; Accepted July 3, 2020

DOI: $10.3892 /$ ijmm.2020.4682

\begin{abstract}
Asthma is a common chronic inflammatory airway disease. Recent studies have reported that interleukin (IL)-33 is a potential link between the airway epithelium and Th2-type inflammatory responses, which are closely related to the progression of asthma. The IL-33 receptor, ST2, is highly expressed in group 2 innate lymphoid cells (ILC2s), Th2 cells, mast cells, eosinophils and natural killer (NK) cells. Cnidii Fructus is a Chinese herb with a long history of use in the treatment of asthma in China. Osthole is one of the major components of Cnidii Fructus. The present study examined the anti-asthmatic effects of osthole in mice and aimed to elucidate the underlying mechanisms involving the IL-33/ST2 pathway. BALB/c mice were sensitized and challenged with ovalbumin and then treated with an intraperitoneal injection of osthole ( 25 and $50 \mathrm{mg} / \mathrm{kg}$ ). Subsequently, the airway hyper-responsiveness (AHR) and inflammation of the lungs were evaluated. The amounts of IL-4, IL-5, IL-13, interferon (IFN) $-\gamma$ and IL-33 in the bronchoalveolar lavage fluid (BALF) were measured by Luminex assay and their mRNA levels in the lungs were measured by reverse transcription-quantitative
\end{abstract}

Correspondence to: Dr Lixin Wang, Department of Integrated Traditional Chinese and Western Medicine, Shanghai Pulmonary Hospital, Tongji University, 507 Zhengmin Road, Yangpu, Shanghai 200433, P.R. China

E-mail: lixinwang99@163.com

Dr Guifang Wang, Department of Respiratory Medicine, Huashan Hospital, Fudan University, 12 Middle Urumqi Road, Xuhui, Shanghai 200040, P.R. China

E-mail: wangguifang@fudan.edu.cn

*Contributed equally

Key words: osthole, asthma, inflammation, interleukin-33, ST2
PCR. The histopathology of the lungs was performed with $\mathrm{H} \& \mathrm{E}, \mathrm{PAS}$ and Masson's staining. The expression of ST2 in the lungs was evaluated by immunohistochemistry. The data demonstrated that osthole markedly reduced AHR and decreased the number of eosinophils and lymphocytes in BALF. It was also observed that osthole significantly inhibited the release of Th2-type cytokines (IL-4, IL-5 and IL-13) and upregulated the IFN- $\gamma$ level in BALF. Moreover, osthole significantly attenuated the IL-33 and ST2 expression in the lungs of asthmatic mice. On the whole, osthole attenuated ovalbumin-induced lung inflammation through the inhibition of IL-33/ST2 signaling in an asthmatic mouse model. These results suggest that osthole is a promising target for the development of an asthma medication.

\section{Introduction}

Allergic asthma is a common chronic inflammatory airway disease. It affects $>300$ million individuals worldwide with increasing prevalence and incidence $(1,2)$. It poses a serious global health concern, resulting in heavy economic burdens on societies worldwide (3). The main pathological characteristics of asthma are airway hyper-responsiveness (AHR), the infiltration of inflammatory cells and mucus overproduction. In addition, long-term airway inflammation leads to structural alterations in the airways.

Type 2 inflammation is one of the major molecular mechanisms underlying susceptibility to asthma. Type 2 immune responses are characteristic of allergic asthma in the lower airways. Airway type 2 immune responses are mainly mediated by eosinophils, basophils, mast cells, Th2 cells, group 2 innate lymphoid cells (ILC2s) and IgE-producing B cells $(4,5)$. Type 2 inflammation is an inflammatory pathway involving a subpopulation of $\mathrm{CD}^{+} \mathrm{T}$ cells. Imbalances between T-helper type 1 (Th1) and T-helper type 2 (Th2) immune reactions are well known to be an underlying factor. In particular, Th2 cells and their cytokines play essential roles in the development of allergic airway inflammation in asthma (6). Briefly, following 
exposure to allergens, the airway epithelium initiates inflammatory reactions involving major pro-inflammatory factors, such as IL-33, IL-25 and thymic stromal lymphopoietin (TSLP), which are cytokines that regulate maturation of $\mathrm{CD}^{+}$ T cells into Th2 cells $(7,8)$. Moreover, Th2 cells release large amounts of Th2 cytokines, such as IL-4, IL-5 and IL-13, while decreasing the production of Th1 cytokines. Type 2 cytokines drive the cellular events in response to the allergen stimulation of the epithelium. These events include activation of the airway epithelial cells, chemoattraction of effector cells (mast cells, eosinophils, and basophils), and remodeling of the epithelium and subepithelial stroma. Such inflammatory responses result in maladaptive changes in the airways, causing pathological reactions to inhaled irritants (9).

IL-33 is a nuclear cytokine belonging to the IL-1 family and is primarily expressed in epithelial and endothelial cells. IL-33 acts as an initiating signal for the inflammatory responses and induces the release of Th2-type cytokines, triggering Th2-cell-mediated immune responses. Recent studies have indicated that the IL-33 level is elevated in asthmatic patients (10). In addition to this clinical observation, IL-33 overproduction has been observed in the lungs of mice in a model of severe influenza-induced asthma exacerbation. This animal model recapitulates all the key features of asthma observed in humans, including the physiological response to treatment with corticosteroids (11).

Allergic asthma immune responses are initiated by the interactions of allergens with epithelial cells, resulting in the release of the cytokines, TSLP, IL-25 and IL-33. IL-25 and IL-33 induce the release of IL-13 and IL-5 from IL-25R $\mathrm{R}^{+}$natural helper cells. IL-33 additionally induces the release of IL-4 from basophils. These responses initiate a pathogenic cascade that leads to the development of asthma in susceptible patients. IL-33 initiates the inflammatory signal transduction pathways via its receptor, ST2, which is mainly expressed in ILC2s, Th2 cells, mast cells, eosinophils, and NK cells (12-14). Recent studies have reported that IL-33 represents a potential link between the airway epithelium and Th2-type inflammatory responses (15-17). Thus, IL-33 appears to be closely involved in the development of asthma. A clinical study demonstrated that the IL-33 level was significantly elevated in the bronchial epithelia of asthmatic patients (18). Accordingly, it has been considered one of the indices of asthma severity $(19,20)$.

The present study investigated the effects of osthole against asthma in mice and it was determined that it ameliorates asthma through the IL-33/ST2 pathway. Currently, the main therapeutic asthma medications include inhaled corticosteroids (ICS), $\beta 2$-agonists and leukotriene modifiers among several others (21). ICS can effectively manage the symptoms of mild to moderate asthma. However, a subgroup of asthma patients chronically treated with ICS gradually become less sensitive, or even resistant, to glucocorticoid. Consequently, the patient becomes susceptible to exaggerated inflammatory responses.

Chinese medicine is currently a major interest in the identification of candidate targets for combinatorial therapy against the pathogenesis of allergic reactions $(22,23)$. Osthole (chemical structure shown in Fig. 1) is a natural compound found in the Chinese herb Cnidii Fructus (She Chuang Zi). This herb has a long history of use for asthma in China. Previous studies have indicated that osthole has a variety of pharmacological and biological activities, including anti-allergic, anti-inflammatory, anti-apoptotic, anti-bacterial and antioxidant stress activities (24-26). Additionally, animal studies have demonstrated that osthole can alleviate immune-mediated inflammatory diseases, such as autoimmune encephalomyelitis, IgA nephropathy, and contact dermatitis (27-29). In line with these physiological responses, osthole has been found to inhibit expression of eotaxin, an IL-4 induced eosinophil-specific C-C chemokine, in bronchial epithelial cells (30).

Therefore, it is suggested that osthole affects IL-33 expression, whereby it regulates the immune response cascade downstream of IL-33, thus providing a possible new treatment option for symptomatic relief medication for allergic asthma. In the present study, the anti-asthmatic effects of osthole in asthmatic mice were investigated.

\section{Materials and methods}

Reagents. Osthole $\left(\mathrm{C}_{15} \mathrm{H}_{16} \mathrm{O}_{3}\right.$; molecular weight, 244.29; purity, $>98 \%$, Fig. 1) was obtained from Winherb. Ovalbumin (OVA; grade $\mathrm{V})$, aluminum hydroxide $\left[\mathrm{Al}(\mathrm{OH})_{3}\right]$, dexamethasone (DEX), methacholine (Mch) and pentobarbital sodium were purchased from Sigma-Aldrich; Merck KGaA. TRIzol reagent and phosphate-buffered saline (PBS) were obtained from Invitrogen; Thermo Fisher Scientific, Inc. SYBR-Green and the PrimeScript 1st Strand cDNA Synthesis kit were supplied by Takara Biotechnology Co., Ltd. The Luminex multiplex cytokine analysis kit was purchased from Merck KGaA. The DAB kit was obtained from Boster Biological Technology Co., Ltd.

Experimental animals and protocol. Female BALB/c mice ( $\mathrm{n}=40 ; 6$ weeks old, weighing 18-20 g) were purchased from Shanghai Xi Puer-Bikai Experimental Animal Co., Ltd. They were housed in a barrier environment free of specific pathogens, and maintained at $22 \pm 2^{\circ} \mathrm{C}$ with $12-\mathrm{h}$ light/dark cycles and free access to standard laboratory food and water. The mice were used for the experiments after 7 days of acclimatization. The experiments were carried out in accordance with the Guide for the Care and Handling of Laboratory Animals of the Institutional Animal Care, and the Ethics Committee of Shanghai Municipal Hospital of TCM, Shanghai University of TCM.

Sensitization and treatment protocol. The BALB/c mice were randomly divided into 5 groups of 8 mice in each as follows: A normal control (NC) group, an OVA-sensitized and challenged asthma model (asthma) group, a dexamethasone $(1 \mathrm{mg} / \mathrm{kg})$ treatment (DEX) group, and 2 osthole treatment groups at doses of 25 and $50 \mathrm{mg} / \mathrm{kg}$ (OS-25 and OS-50). Apart from the mice in the $\mathrm{NC}$ group, the mice in all the other groups were sensitized by an intraperitoneal injection of $20 \mu \mathrm{g}$ OVA together with $2 \mathrm{mg}$ of $\mathrm{Al}(\mathrm{OH})_{3}$ in $200 \mu \mathrm{l}$ of PBS on days 1, 7, 14 and 21. The mice were then sprayed with $2 \%$ OVA (w/v in PBS) for 30 min each day from day 24 to day 30, as previously described (31). Briefly, the mice were placed in a transparent box that was connected to the atomization tube of an ultrasonic nebulizer, and atomized with OVA (402AI, Jiangsu Yuyue Medical Instrument Co., Ltd.). PBS was used instead of OVA for the NC group. From days 24 to 30, the mice in the drug intervention groups were intraperitoneally administered osthole ( 25 or $50 \mathrm{mg} / \mathrm{kg}$ ) or dexamethasone 


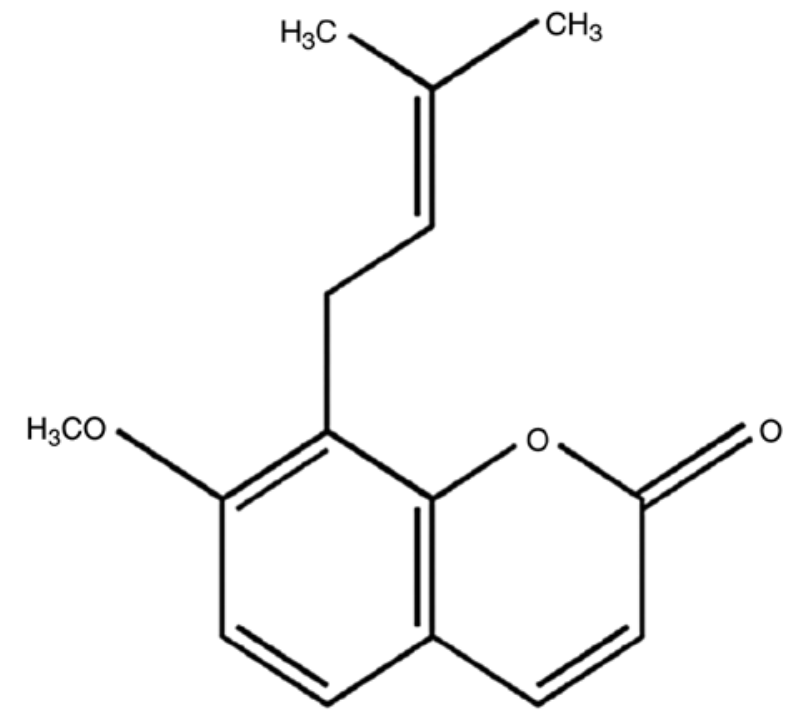

Figure 1. Chemical structure of osthole (7-methoxy-8-prenyl coumarin).

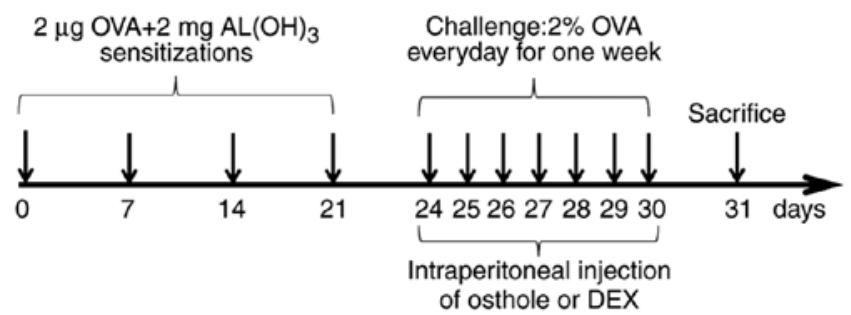

Figure 2. Experimental protocol for OVA-induced asthma murine model. BALB/c mice were sensitized with OVA and aluminum hydroxide by 4 intraperitoneal injections on days I, 7, 14 and 21. Then, mice were sprayed with $2 \%$ OVA (w/v in PBS) for 30 min each day from day 24 to day 30 . The normal control group used PBS instead of OVA to sensitize and challenge. Dexamethasone and osthole were administered via intraperitoneal injection $1 \mathrm{~h}$ before the challenge from day 24 to 30 . Mice were sacrificed within $24 \mathrm{~h}$ after the final OVA challenge. OVA, ovalbumin; DEX, dexamethasone.

$(1 \mathrm{mg} / \mathrm{kg}) 1 \mathrm{~h}$ prior to the OVA atomization. Osthole and DEX treatments were administered daily where indicated. The mice in the NC and asthma groups were administered PBS only at the same volume used for drug administration. Subsequently, the animals were anesthetized by an intraperitoneal injection of pentobarbital sodium $(50 \mathrm{mg} / \mathrm{kg})$ at $24 \mathrm{~h}$ after the final challenge for AHR detection. A schematic diagram of the airway inflammation induction is presented in Fig. 2.

AHR detection. Bronchial hyperreactivity was measured $24 \mathrm{~h}$ after the final challenge. AHR was detected using an invasive, mouse pulmonary function testing system (Buxco Electronics Inc.). The entire procedure was performed on anesthetized mice. First, the mice were anesthetized with pentobarbital sodium $(50 \mathrm{mg} / \mathrm{kg}$, intraperitoneal injection), followed by tracheotomy, after which a suitable cannula was inserted. The mice were then placed in the plethysmograph chamber, and the ventilator-assisted mouse breathing tube was opened while increasing the airway pressure baseline with $<5 \%$ change every $2.5 \mathrm{~min}$ until it tended to stabilize. Subsequently, PBS and 3 concentrations of Mch $(3.125,6.25$ and $12.5 \mathrm{mg} / \mathrm{ml})$ were introduced into the head chamber via nebulization. The airway responses were displayed through changes in airway resistance (RL) and lung dynamic compliance (Cdyn) (32). The dose-dependent effect of osthole (25 and $50 \mathrm{mg} / \mathrm{kg}$ ) on airway resistance in asthmatic mice was evaluated by statistical analysis. If the inhibitory effect increased with increasing osthole concentration, the inhibitory effect of osthole on airway resistance was considered to be dose-dependent (33-35).

Collection of bronchoalveolar lavage fluid (BALF). After the airway reactivity test was completed, the mice were euthanized by cervical dislocation, and death was confirmed on the basis of lack of breathing, pulse, pupillary reflex, corneal reflex and response to firm toe pinching. The lungs were lavaged through the tracheal cannula 3 times with $0.8 \mathrm{ml}$ cold PBS. The BALF was then collected and centrifuged at $500 \mathrm{xg}$ for $10 \mathrm{~min}$ at $4^{\circ} \mathrm{C}$. The supernatants were transferred into new tubes and stored at $-80^{\circ} \mathrm{C}$. The cell pellets were resuspended in PBS to evaluate the abundance of inflammatory cells, including lymphocytes, eosinophils, basophils and neutrophils. The cytokine (INF- $\gamma$, IL-4, IL-5, IL-13 and IL-33) levels in BALF were analyzed by Luminex assay following the manufacturer's instructions.

Histopathology of lung tissue assay. The right middle lung lobes were removed from the mice and fixed with $4 \%$ paraformaldehyde. First, the lungs were dehydrated with various concentrations of alcohol. They were then embedded in paraffin and sectioned at a thickness of $3 \mu \mathrm{m}$. Subsequently, the sections were stained with hematoxylin and eosin (H\&E; cat. no. AR1180; Boster Biological Technology, Ltd.) for 5 min, periodic acid-Schiff reagent (PAS; cat. no. BA4080B; BaSO Biotech) for $10 \mathrm{~min}$, and Masson's trichrome (M-T; cat. no. BA4079B; BaSO Biotech) for $10 \mathrm{~min}$ at room temperature. The lung tissue sections were examined at x200 magnification using an optical microscope (ECLIPSE 80i, Nikon Corp.). Finally, lung inflammation was assessed by the degree of eosinophil infiltration, the amount of mucus secretion and the area of collagen deposition.

$q P C R$ assay. Reverse transcription-quantitative PCR (RT-qPCR) was performed to determine the levels of the following Th1/Th2-related cytokines in the lungs: IFN- $\gamma$, IL-4, IL-5, IL-13, IL-33 and ST2. Total RNA was extracted from the lungs using TRIzol reagent. A reverse transcriptase kit was used to perform first-strand cDNA synthesis according to the manufacturer's protocol. The cDNA samples were then amplified using an ABI 7500 sequence detector with thermal cycling conditions of $30 \mathrm{sec}$ at $95^{\circ} \mathrm{C}$ (hot-start step), followed by 40 cycles of $5 \mathrm{sec}$ at $95^{\circ} \mathrm{C}$ (denaturation step), $60 \mathrm{sec}$ at $60^{\circ} \mathrm{C}$ (annealing step) and $60 \mathrm{sec}$ at $72^{\circ} \mathrm{C}$ (extension step). Relative mRNA expression was analyzed using the $2^{-\Delta \Delta \mathrm{Cq}}$ method (36). $\beta$-actin was used as the internal control gene. The sequences of the primers used for mouse $\beta$-actin, IL-4, IL-5, IL-13, IFN- $\gamma$, IL-33 and ST2 are presented in Table I.

Immunohistochemistry (IHC). ST2 expression in the lungs was evaluated by IHC according to the manufacturer's instructions (ProteinTech Group, Inc.). First, the lung sections were deparaffinized and washed 3 times with PBS. They were then incubated in $3 \%$ hydrogen peroxide for $30 \mathrm{~min}$. Subsequently, they were washed with PBS, and incubated with rabbit polyclonal anti-ST2 
Table I. Primer sequences of the genes detected by quantitative polymerase chain reaction.

\begin{tabular}{ll}
\hline Gene & \multicolumn{1}{c}{ Primer sequences (5'-3') } \\
\hline$\beta$-actin & F: 5'-CCTCTATGCCAACACAGT-3' \\
& R: 5'-AGCCACCAATCCACACAG-3' \\
$I L-4$ & F: 5'-TAGTTGTCATCCTGCTCTTCTT-3' \\
& R: 5'-CTCACTCTCTGTGGTGTTCTTC-3' \\
$I L-5$ & F: 5'-CCATTGCCCACTCTGTAC-3' \\
& R: 5'-AGGCTTCCTGTCCCTACT-3' \\
$I L-13$ & F: 5'-CAGCCTCCCCGATACCAAAAT-3' \\
& R: 5'-CCCCAGCAAAGTCTGATGTGA-3' \\
$I F N-\gamma$ & F: 5'-CAGCGACCGTGTCTGTAT-3' \\
$I L-33$ & R: 5'-GAGGAGCGTCTGGAAATA-3' \\
& F: 5'-GTCAACAGACGCAGCAAA-3' \\
$S T 2$ & R: 5'-TTAGGAAAGAACCCACGAA-3' \\
& F: 5'-CTGGCACTGCATTTCCT-3' \\
& R: 5'-GCCTACGAGCAGGAGATT-3'
\end{tabular}

antibody (1:100; cat. no. 11920-1-AP, ProteinTech Group, Inc.) for $12 \mathrm{~h}$ at $4^{\circ} \mathrm{C}$ in a humid chamber. The sections were washed with PBS, covered with biotinylated goat anti-rabbit (1:300; cat. no. SA1022, Wuhan Boster Biological Technology,Ltd.), and incubated for $60 \mathrm{~min}$ at room temperature. Once the incubation was completed, they were washed again with PBS, incubated in a peroxidase solution for $20 \mathrm{~min}$ at room temperature, and then washed again with PBS. Finally, the sections were covered with 3,3-diaminobenzidine tetrahydrochloride (DAB) chromogen and incubated for 3-10 $\mathrm{min}$ at room temperature before being observed under a light microscope (ECLIPSE 80i, Nikon Corp.) at X200 magnification.

Statistical analysis. All data are presented as the means \pm standard deviation. Data analysis was performed using SPSS version 20.0 (IBM SPSS, Inc.). The statistical graphs were created using GraphPad Prism 6.0 software (GraphPad Software, Inc.). Results were evaluated statistically using one-way analysis of variance (ANOVA) and the Bonferroni post hoc test. Values of $\mathrm{P}<0.05$ and $\mathrm{P}<0.01$ were considered to indicate statistically significant and highly statistically significant differences, respectively.

\section{Results}

Osthole reduces AHR in asthmatic mice. The effects of osthole on airway responses in a mouse model of asthma was determined by nebulization with Mch. A higher level of airway resistance was observed in the mice with OVA-induced asthma compared with that observed in the normal control group $(\mathrm{P}<0.05)$, whereas DEX and osthole treatment significantly downregulated the OVA-induced AHR $(\mathrm{P}<0.05$; Fig. 3). In particular, osthole exerted a more prominent effect at $50 \mathrm{mg} / \mathrm{kg}$ than that observed with $25 \mathrm{mg} / \mathrm{kg}$.

Osthole attenuates airway inflammation in asthmatic mice. Inflammatory cell infiltration is one of the most prominent

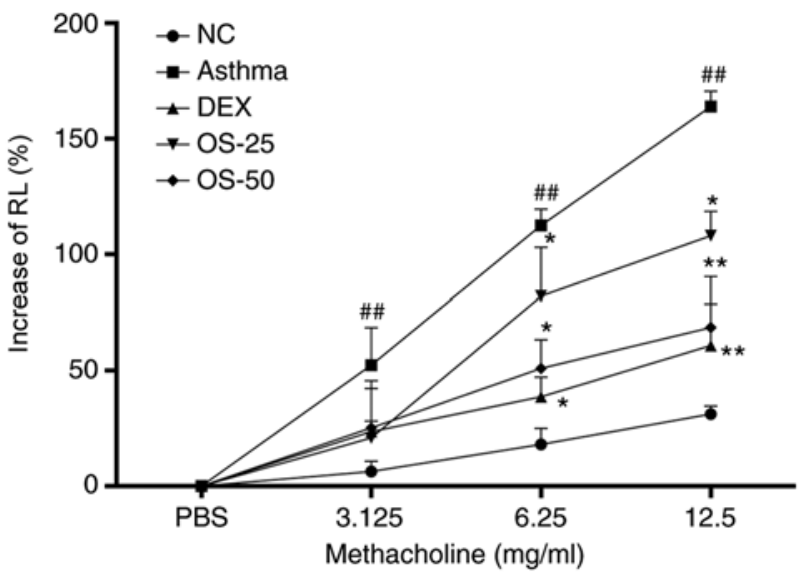

Figure 3. Osthole reduces AHR in asthmatic mice. Effect of osthole on AHR in response to increasing concentrations of aerosolized methacholine $(3.125,6.25$ and $12.5 \mathrm{mg} / \mathrm{ml})$ or PBS $24 \mathrm{~h}$ after the final OVA challenge for mice. AHR was assessed by lung resistance (RL). The normal control group (NC), OVA sensitized and challenged asthma group (asthma), dexamethasone $(1 \mathrm{mg} / \mathrm{kg})$ treatment group (DEX), and osthole treatment groups at doses of 25 and $50 \mathrm{mg} / \mathrm{kg}$ (OS-25 and OS-50). The data are presented as the mean \pm SD. ${ }^{\# \#} \mathrm{P}<0.01$ vs. the normal control group; ${ }^{*} \mathrm{P}<0.05,{ }^{* *} \mathrm{P}<0.01$ vs. the asthma model group. OVA, ovalbumin; DEX, dexamethasone; AHR, airway hyper-responsiveness.

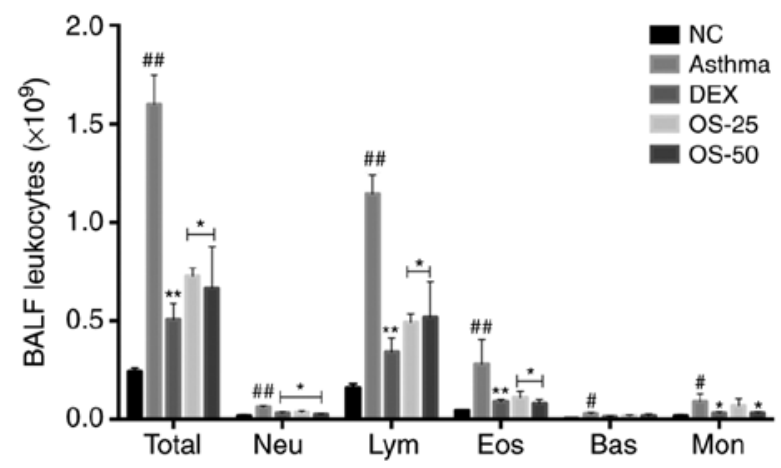

Figure 4. Osthole reduces the number of inflammatory cells in the alveolar lavage fluid of asthmatic mice. Total, leucocytes; eos, eosnophils; lym, lymphocytes; neu, neutrophils; bas, basophils; mon, monocytes. The normal control group (NC), OVA-sensitized and challenged asthma group (asthma), dexamethasone $(1 \mathrm{mg} / \mathrm{kg})$ treatment group (DEX), and osthole treatment groups at doses of 25 and $50 \mathrm{mg} / \mathrm{kg}$ (OS-25, OS-50). The data are presented as the means $\pm \mathrm{SD} .{ }^{\#} \mathrm{P}<0.05,{ }^{\# \#} \mathrm{P}<0.01$ vs. the normal control group; ${ }^{*} \mathrm{P}<0.05$, ${ }^{* * *} \mathrm{P}<0.01$ vs. the asthma model group. OVA, ovalbumin; DEX, dexamethasone.

features of asthma. The total and differential cell counts of BALF were estimated with an automated cell counter. The numbers of total leukocytes and differential cells, including neutrophils, eosinophils, lymphocytes, basophils and monocytes were markedly increased in the asthma group compared with those of the NC group ( $\mathrm{P}<0.05$; Fig. 4). After the mice were treated with osthole or DEX, the numbers of leukocytes and differential cells markedly decreased $(\mathrm{P}<0.05)$. In addition, osthole treatment decreased the number of eosinophils in a dose-dependent manner. It was clearly indicated that the optimal dose of osthole with which to reduce the number of inflammatory cells was $50 \mathrm{mg} / \mathrm{kg}(\mathrm{P}<0.05)$. H\&E staining also revealed that there were more inflammatory cells infiltrated in the lungs of mice in the asthmatic group (Fig. 5B) compared with those in the lungs of mice in the NC group (Fig. 5A). 

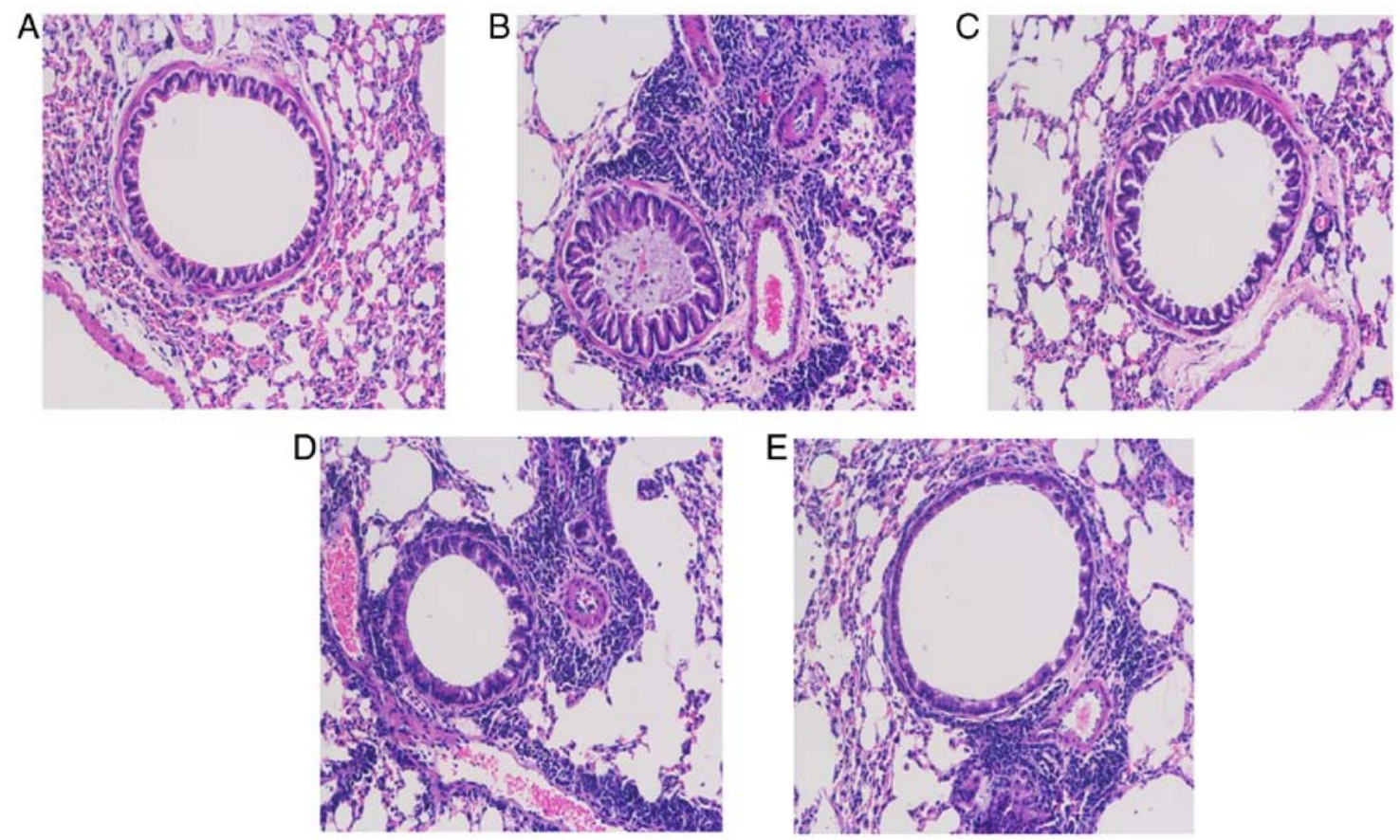

Figure 5. Osthole alleviates airway inflammation in the lungs of mice with OVA-induced asthma (H\&E staining, $\mathrm{x} 200 \mathrm{magnification})$. The images show the infiltration of inflammatory cells. (A) normal control group, (B) asthma mice model, (C) DEX treatment group, (D) osthole treatment group (25 mg/kg), (E) osthole treatment group (50 mg/kg). OVA, ovalbumin; DEX, dexamethasone.
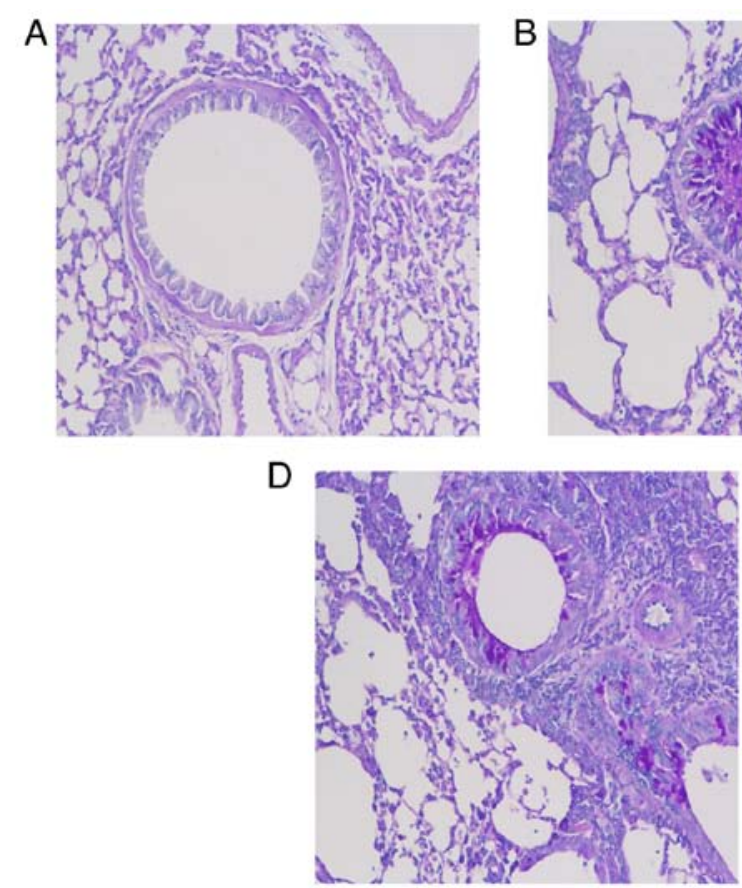
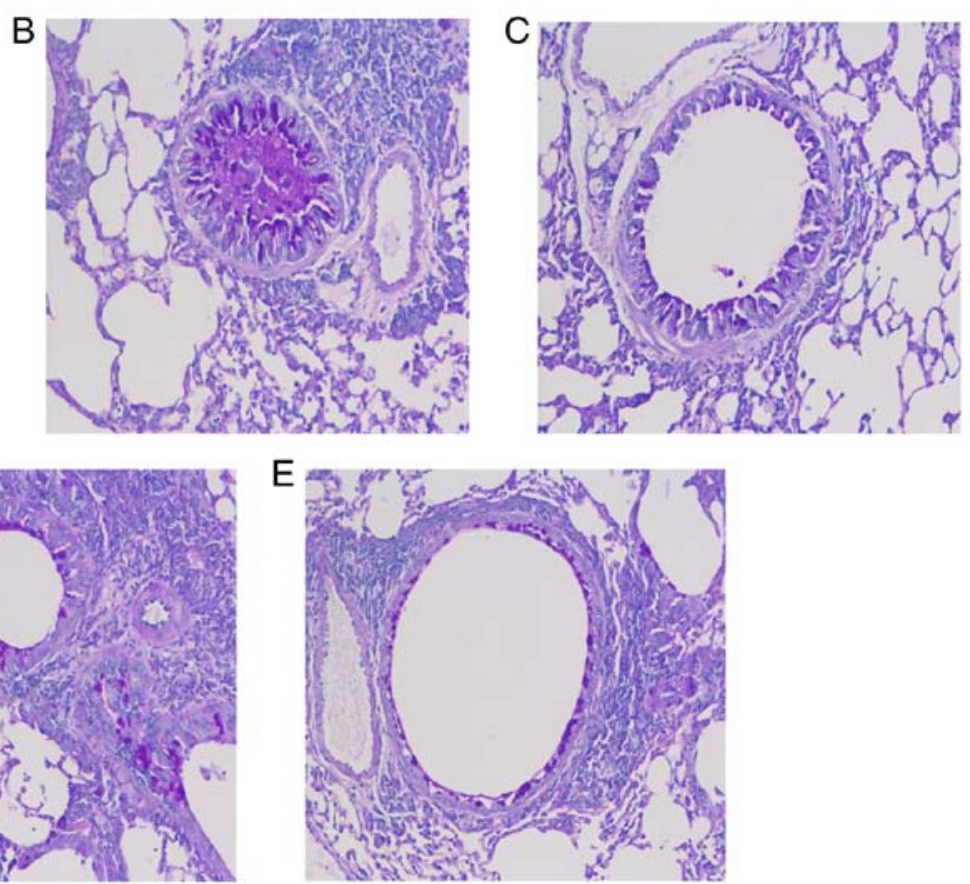

Figure 6. Oshtole decreases goblet cell metaplasia. Goblet cell hyperplasia was estimated with PAS staining (x200 magnification). (A) normal control group, (B) asthma mice model, (C) DEX treatment group, (D) osthole treatment group ( $25 \mathrm{mg} / \mathrm{kg}),(\mathrm{E})$ osthole treatment group (50 mg/kg). OVA, ovalbumin; DEX, dexamethasone.

However, airway inflammation was alleviated in the OS and DEX groups (Fig. 5C-E).

Osthole alleviates airway remodeling in asthmatic mice. The degree of airway remodeling is associated with the severity of asthma. In the present study, PAS and Masson's trichrome staining were used to assess mucus secretion and collagen deposition in addition to the presence of goblet cell hyperplasia (Figs. 6 and 7). Compared with the NC group, the mice in the asthma group exhibited increased mucus production, excessive collagen deposition and goblet cell hyperplasia in the lungs (Figs. 6A and B, and 7A and B), and these maladaptive changes were suppressed in the DEX and OS groups (Figs. 6C-E and 7C-E). Of note, no marked 

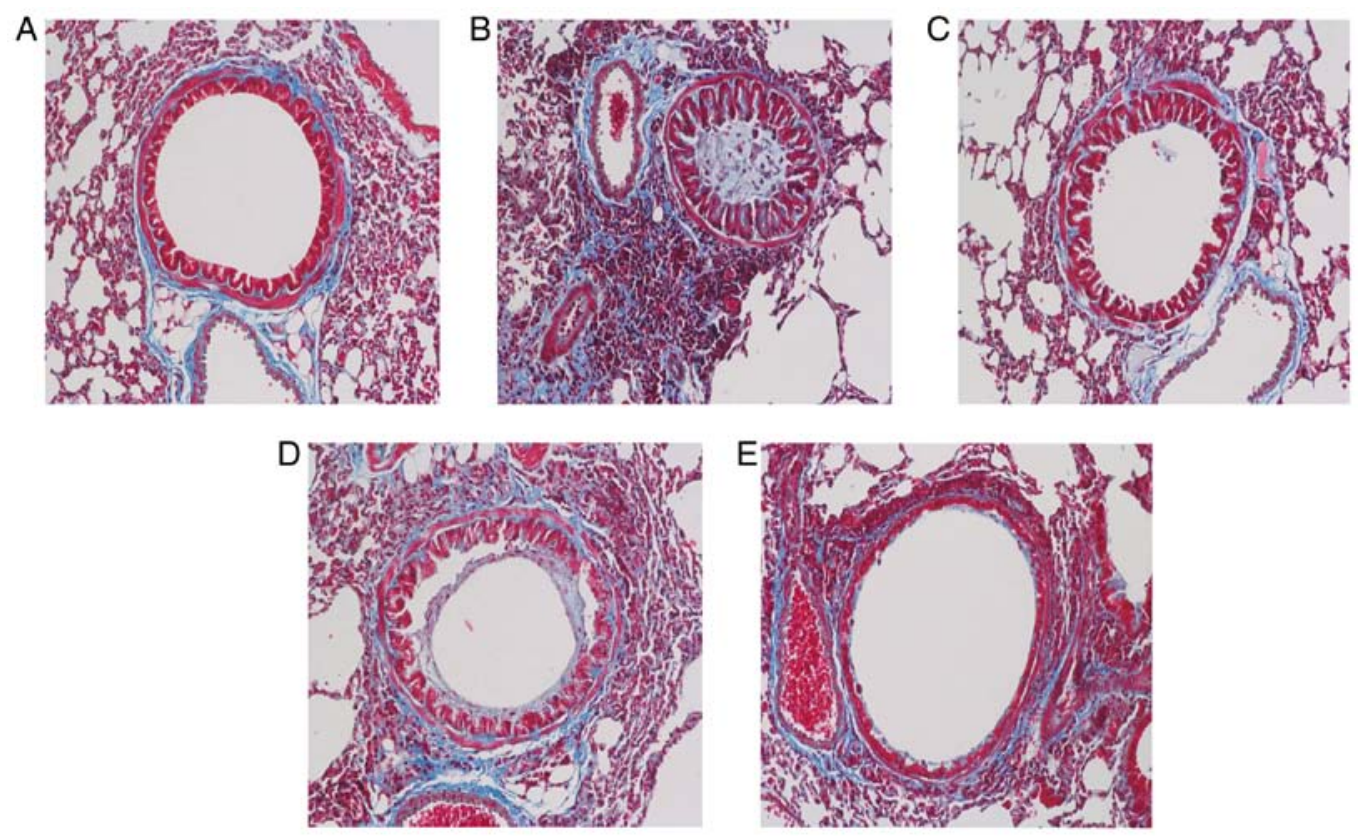

Figure 7. Oshtole inhibits collagen deposition. collagen deposition was examined by Masson's trichrome staining (x200 magnification). (A) normal control group, (B) asthma mice model, (C) DEX treatment group, (D) osthole treatment group (25 mg/kg), (E) osthole treatment group (50 mg/kg). OVA, ovalbumin; DEX, dexamethasone.

differences were observed between the 2 drug treatment groups (Fig. 7C-E).

Osthole rebalances Th1/Th2-related cytokines in the BALF and lungs of asthmatic mice. Subsequently, the present study evaluated the levels of the Th1/Th2-related cytokines, IFN- $\gamma$, IL-4, IL-5 and IL-13, in BALF using a Luminex multiplex cytokine analysis kit. The levels of the Th2 cytokines, IL-4, IL-5 and IL-13, were significantly elevated in the asthma group $(\mathrm{P}<0.05)$; however, these elevations were markedly downregulated in the OS-50 and DEX groups ( $\mathrm{P}<0.05$; Fig. 8B-D). At the same time, the level of the Th1 cytokine, IFN- $\gamma$, was decreased in the asthma group $(\mathrm{P}<0.05)$, while the DEX and OS groups exhibited a relatively higher level of IFN $-\gamma(\mathrm{P}<0.05$; Fig. 8A). Notably, the level of IFN- $\gamma$ in the OS-50 group was much higher than that in the DEX group (Fig. 8A). Furthermore, the mRNA levels of these cytokines were also evaluated in the lungs by RT-qPCR. The IFN- $\gamma$ mRNA levels in the OS and DEX groups were higher than those in the asthma group $(\mathrm{P}<0.05)$, while the IL-4, IL-5 and IL-13 mRNA levels were lower in the OS and DEX groups $(\mathrm{P}<0.05)$. Notably, the effect of osthole $(50 \mathrm{mg} / \mathrm{kg})$ on IFN- $\gamma$ mRNA expression was much more prominent than that of DEX (Fig. 8E).

Osthole inhibits IL-33/ST2 signaling in asthmatic mice. IL-33/ST2 signaling is involved in airway inflammation in asthma. The level of IL-33 in BALF was assessed using the Luminex multiplex cytokine analysis kit. Moreover, the protein level of ST2 was evaluated by IHC. Furthermore, the mRNA levels of IL-33 and ST2 in the lungs were quantified by RT-qPCR. The levels of IL-33 in BALF of in the asthma group were higher than those in the control group $(\mathrm{P}<0.05$; Fig. 9F). On the other hand, the OS-50 and DEX groups exhibited significantly lower levels of IL-33 than the asthma group.
In terms of gene expression, there was a significant upregulation in the IL-33 and ST2 mRNA levels in the asthma group compared with the NC group $(\mathrm{P}<0.05)$. Of note, the DEX and OS groups exhibited significantly lower mRNA levels of IL-33 and ST2 compared with the asthma group $(\mathrm{P}<0.05$; Fig. 9G and H). Moreover, the effect of osthole treatment appeared to be dose-dependent, since the IL-33 and ST2 mRNA levels were markedly lower in the OS-50 group relative to those in the OS-25 group. Furthermore, the IHC analysis of the lung sections demonstrated that the highest ST2 protein expression was observed in the asthma group. By constast, the DEX and OS-50 groups exhibited lower amounts of ST2 protein (Fig. 9A-E).

\section{Discussion}

AHR is a representative hallmark of allergic asthma. It can be induced by exposure to allergens, such as OVA and house dust mite (HDM) (37-39). Osthole is a natural compound isolated from the Chinese herb, Cnidii Fructus, which has been widely used for centuries in China for the treatment of allergic disorders. In the present study, it was demonstrated for the first time, at least to the best of our knowledge, that osthole treatment can markedly decrease AHR in a mouse model of asthma.

Allergic asthma is a chronic airway inflammatory disorder. It is considered to manifest from the immune responses of Th2 cells, including innate lymphoid cells (ILC2s), CD4 ${ }^{+} \mathrm{Th} 2$ cells, eosinophils, neutrophils and mast cells. These maladaptive immune responses are executed via the overproduction of the IL-4, IL-5 and IL-13 cytokines. IL-4 and IL-5 promote the recruitment of inflammatory cells. For example, IL-5 stimulates the generation and activation of eosinophils. In the present study, the types of inflammatory cells in BALF were analyzed. It was observed that the number of leucocytes, particularly eosinophils, was markedly elevated in the 

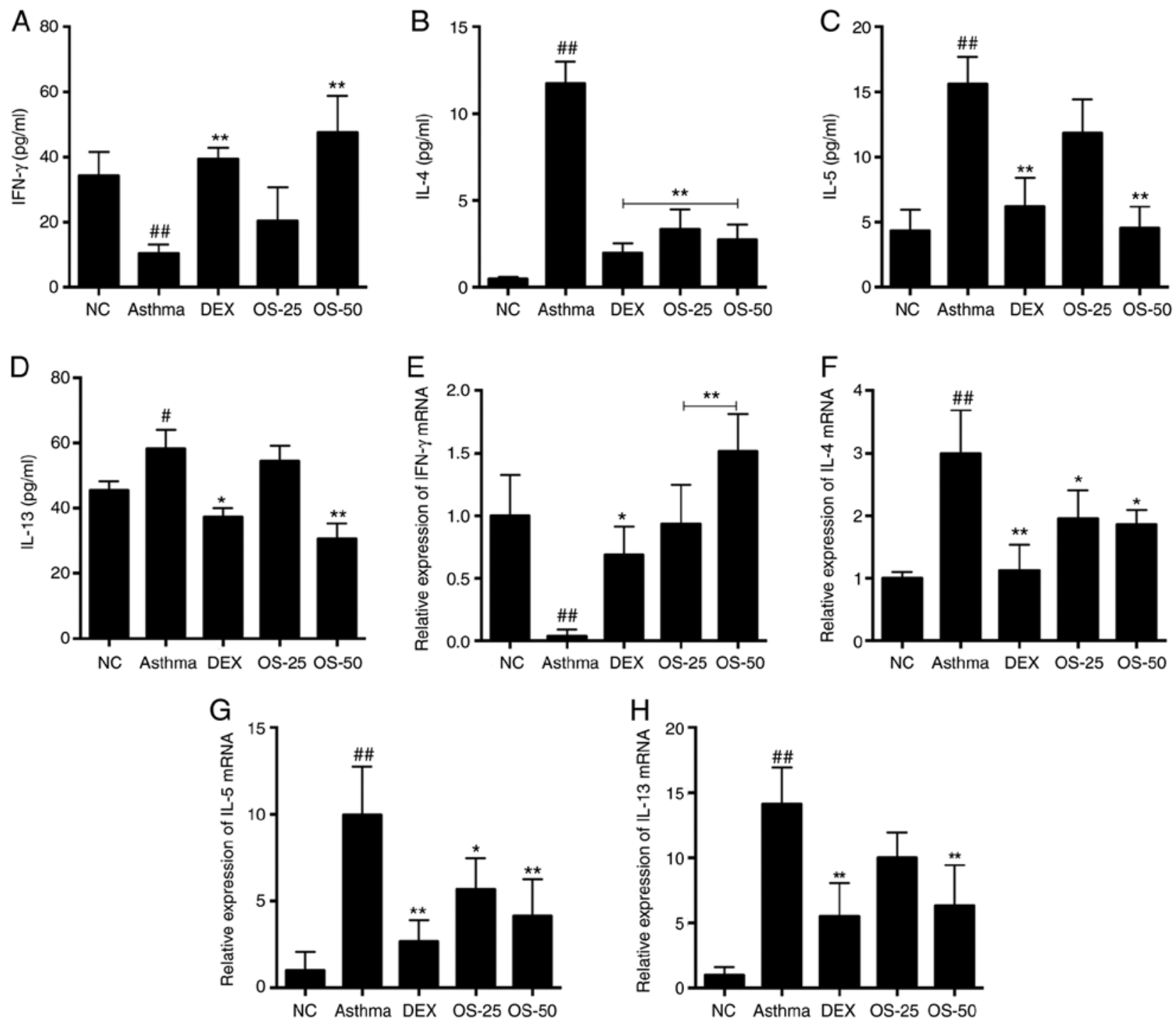

Figure 8. (A-D) Osthole suppresses the secretion of Th2 cytokines, and promotes the production of Th1 cytokines in BALF. The levels of IFN- $\gamma$, IL-4, IL-5 and IL-13 were measured using a Luminex multiplex cytokine analysis kit. (E-H) The mRNA levels of IFN- $\gamma$, IL-4, IL-5 and IL-13 in lung tissues. Results are expressed as the means $\pm \mathrm{SD} .{ }^{\#} \mathrm{P}<0.05,{ }^{\# \#} \mathrm{P}<0.01$ vs. the normal control group; ${ }^{*} \mathrm{P}<0.05,{ }^{* *} \mathrm{P}<0.01$ vs. the asthma model group. OVA, ovalbumin; DEX, dexamethasone.

asthmatic mice as was expected, confirming that the asthmatic model in the present study recapitulated the major features of asthma observed in humans. Notably, the osthole-treated asthmatic mice exhibited lower amounts of leucocytes, including eosinophils, indicating that osthole treatment reduced the number of inflammatory cells. Furthermore, it was found that the levels of IL-4, IL-5 and IL-13 were increased in the BALF of asthmatic mice, while the level of IFN- $\gamma$ was decreased. Osthole treatment partially suppressed the imbalance between these Th1- and Th2-related cytokines. IL-13 has been reported to induce smooth muscle cell proliferation and hypertrophy, goblet cell metaplasia, the overproduction of mucus and excessive collagen deposition (40). The present study also observed that the asthmatic mice had significantly less mucus and goblet cells when treated with osthole.

Epithelial cells (ECs) are the first line of defense against pathogens. They also interact with a various types of immune cells that trigger immune responses against foreign pathogens. Most importantly, ECs have been reported to affect Th2 responses by secreting the pro-inflammatory cytokines, IL-33, IL-25 and TSLP (41). IL-33 is a newly discovered member of the IL-1 family (42). It is mainly located in the cell nucleus in addition to its extra-cellular role as a cytokine $(43,44)$. Genome-wide association studies have indicated that IL-33 is one of the genes involved in asthma development $(45,46)$. IL-33 is constitutively expressed in epithelial, endothelial, and mast cells $(47,48)$. IL-33 is rapidly released form epithelial cells after allergen exposure or tissue injury $(13,49)$. IL-33 can function as an alarm to warn the immune system. IL-33 signals activated via its receptor ST2, which is expressed on natural helper cells, ILC2s, eosinophils, basophils, Th2 cells, mast cells and NK cells $(50,51)$. Previous studies have revealed that IL-33/ST2 signaling is the fundamental Th2-associated immune response $(12,52,53)$. The inhibition of IL-33 expression reduces the symptoms of asthma, such as inflammation, in asthmatic mice (54). In the present study, it was identified that IL-33 and ST2 levels were significantly elevated in the lungs of asthmatic mice; however, this inflammatory response 

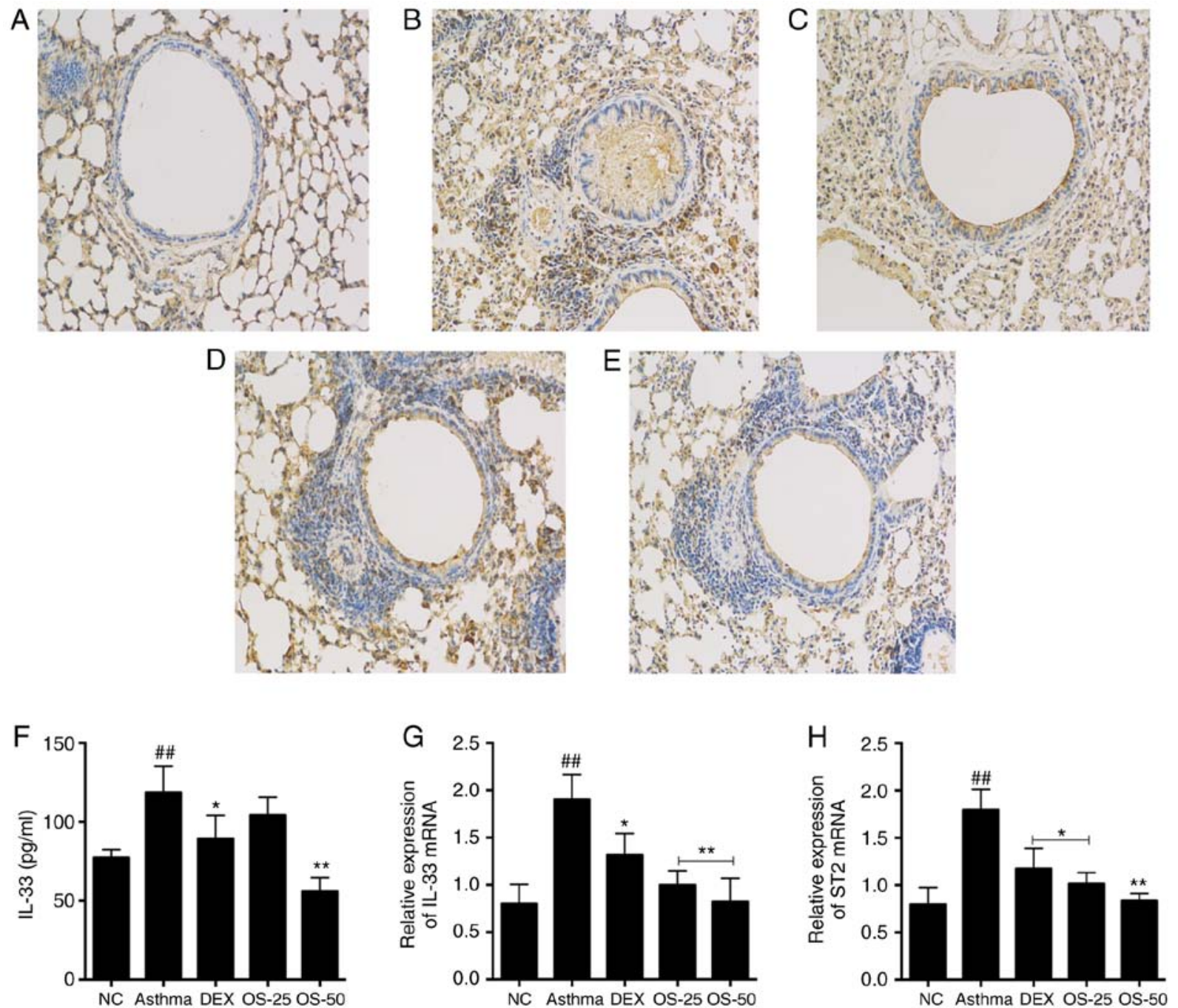

Figure 9. (A-E) Expression of ST2 is increased in lung tissue of asthmatic mice by immunohistochemistry. The ST2 immunostaining (brown stain) at $\mathrm{x} 200$ magnification is shown. (A) normal control group, (B) asthma mice model, (C) DEX treatment group, (D) osthole treatment group (25 mg/kg), (E) osthole treatment group $(50 \mathrm{mg} / \mathrm{kg}$ ). (F) The secretion of IL-33 in BALF was assessed using the Luminex multiplex cytokine assat kit. (G) Relative expression of IL-33 mRNA in lung. (H) Relative expression of ST2 mRNA in lung. Values were expressed as the means $\pm \mathrm{SD}$. ${ }^{\#} \mathrm{P}<0.01$ vs. the normal control group; ${ }^{*} \mathrm{P}<0.05$, ${ }^{* *} \mathrm{P}<0.01$ vs. the asthma model group. OVA, ovalbumin; DEX, dexamethasone.

was significantly suppressed by osthole treatment. Further studies are required to determine exactly which cells mediate IL-33/ST2 signaling and to elucidate the mechanisms through which they interact with other cells.

Osthole has a long history of clinical application in the treatment of dermatitis and eczema. Although it has a variety of biological activities, data on its efficacy on allergic asthma is limited. In the present study, the immunomodulatory effects of osthole in asthmatic mice were investigated. Osthole did not exert an adverse side-effects when administered at 25 or $50 \mathrm{mg} / \mathrm{kg}$ in mice. The results indicated that exposure to OVA induced allergic asthma in mice and that osthole significantly reduced airway resistance, and the infiltration of the above-mentioned inflammatory cells. In summary, the data suggest that osthole can alleviate allergen-induced airway inflammation in mice.

Th2-type cytokines play a key role in the progression of allergic asthma and can promote the development of inflammatory phenotypes. IL-4 is an important inducer of IgE production by B lymphocytes, and IL-5 promotes eosinophil activation, maturation and recruitment. IL-13 is more effective in promoting AHR and pathological changes. IL-33 stimulates excessive secretion of Th2 cytokines. The treatment of asthmatic mice with high doses of osthole $(50 \mathrm{mg} / \mathrm{kg})$ significantly reduced the expression of the Th2 cytokines, IL-4, IL-5 and IL-13. Overall, these data indicate that osthole can attenuate OVA-induced Th2 responses and inhibit the immunopathology of allergic asthma in mice. Based on the aforementioned information, treatment strategies for the pain suppression of Th2 immunity may improve the control of allergic asthma. Osthole improves the severity of allergic asthma in mice by inhibiting the Th2 immune response. The present study further elucidated whether this inhibitory effect was in part due to IL-33 downregulation in osthole-treated mice. The results revealed that IL-33 production was significantly decreased in the lungs and BALF. Likewise, it was observed that the IL-33 ligand, ST2, was also significantly downregulated in the lungs.

In the present study, OVA was used to induce asthma in mice, whereby it was observed that osthole reduced the degree of inflammation in asthma. In the future, the authors aim to 
evaluate the effects of osthole on allergic asthma induced by other allergens, such as HDM.

IL-33 has been reported to trigger the activation of inflammatory pathways through an IL-1-related receptor protein complex of ST2 (ST2/IL-1RAcP) (20). Since dendritic cells (DCs) function as antigen-presenting cells, they play a central role in immune responses observed in asthma. The activation of Toll-like receptor 4 (TLR4) by a fungal proteinase derived from Aspergillus oryzae has been reported to be involved in the epithelial overexpression of IL-33 in the airways, subsequently activating DCs (55). Moreover, HDM extracts can directly activate ECs to produce IL-25 and IL-33 and act on DCs. Thus, ECs appear to modulate DC activation through secretion of IL-33 (56). Given this link between ECs and DCs, further studies are required to investigate the role of DCs in the osthole-induced alleviation of airway inflammation in asthmatic mice.

There are several other limitations to the present study. It is not clear which cell types were affected by Osthole, resulting in reduced airway inflammatory responses. Osthole may play a regulatory role in airway epithelial cells and dendritic cells, which in turn affects the Th2 immune response process, leading to a reduction in airway inflammation. The intervention of osthole in airway epithelial cells and DCs is the content of follow-up experiments by the authors.

In conclusion, the results of the present study indicate that osthole administration reduces the severe symptoms of Th2-mediated asthma in mice, in part as it reduces the expression of IL-33. The data demonstrate that osthole treatment suppresses Th2 immune responses by inhibiting the production and activation of IL-33 in the early stages of antigen stimulation. Furthermore, it was determined that Th1 and Th2 cells were rebalanced presumably through the inhibition of IL-33/ST2 signaling. Taken together, the results indicate that osthole may be a promising candidate for the development of an asthma medication.

\section{Acknowledgements}

The authors would like to thank Dr Jinfeng Wu (Huashan Hospital, Fudan University)for his critical reading of the manuscript. The authors also gratefully acknowledge the expert technical assistance provided by Zhonghua Wu (Shanghai University of Traditional Chinese Medicine) and Xiaoming Wang (Shanghai University of Traditional Chinese Medicine).

\section{Funding}

The present study was funded by a grant from the National Natural Science Foundation of China (grant no. 81573937).

\section{Availability of data and materials}

The datasets used or analyzed during the current study are available from the corresponding author on reasonable request.

\section{Authors' contributions}

QY,LK,WH, NM, XL, GW and LW were involved in the conceptualization of the study. QY and LK performed the experiments, contributed to data analysis and wrote the manuscript. GW and LW conceptualized the study design. QY, LK, WH, NM and XL contributed to data analysis and provided experimental materials. All authors have read and approved the final manuscript.

\section{Ethics approval and consent to participate}

All animal experiments were approved by the Committee on the Ethics of Animal Experiments of Shanghai Municipal Hospital of TCM, Shanghai University of TCM.

\section{Patient consent for publication}

Not applicable.

\section{Competing interests}

The authors declare that they have no competing interests.

\section{References}

1. Bostantzoglou C, Delimpoura V, Samitas K, Zervas E, Kanniess F and Gaga M: Clinical asthma phenotypes in the real world: Opportunities and challenges. Breathe (Sheff) 11: 186-193, 2015.

2. Galli SJ, Tsai M and Pilponsky AM: The development of allergic inflammation. Nature 454: 445-454, 2008.

3. Bateman ED, Hurd SS, Barnes PJ, Bousquet J, Drazen JM, FitzGerald M, Gibson P, Ohta K, O'Byrne P, Pedersen SE, et al: Global strategy for asthma management and prevention: GINA executive summary. Eur Respir J 31: 143-178, 2008.

4. Oliphant CJ, Barlow JL and McKenzie AN: Insights into the initiation of type 2 immune responses. Immunology 134: 378-385, 2011.

5. Fahy JV: Type 2 inflammation in asthma-present in most, absent in many. Nat Rev Immunol 15: 57-65, 2015.

6. Martinez FD and Vercelli D: Asthma. Lancet 382: 1360-1372, 2013.

7. Hwang YH, Paik MJ and Yee ST: Diisononyl phthalate induces asthma via modulation of Th1/Th2 equilibrium. Toxicol Lett 272: 49-59, 2017.

8. Platts-Mills TA: The allergy epidemics: 1870-2010. J Allergy Clin Immunol 136: 3-13, 2015.

9. Locksley RM: Asthma and allergic inflammation. Cell 140: 777-783, 2010.

10. Smith DE: IL-33: A tissue derived cytokine pathway involved in allergic inflammation and asthma. Clin Exp Allergy 40: 200-208, 2010.

11. Ravanetti L, Dijkhuis A,SabogalPineros YS,Bal SM,DierdorpBS, Dekker T, Logiantara A, Adcock IM, Rao NL, Boon L, et al: An early innate response underlies severe influenza-induced exacerbations of asthma in a novel steroid-insensitive and anti-IL-5-responsive mouse model. Allergy 72: 737-753, 2017.

12. Saluja R, Ketelaar ME, Hawro T, Church MK, Maurer M and Nawijn MC: The role of the IL-33/IL-1RL1 axis in mast cell and basophil activation in allergic disorders. Mol Immunol 63: 80-85, 2015.

13. Kaur D, Gomez E, Doe C, Berair R, Woodman L, Saunders R, Hollins F, Rose FR, Amrani Y, May R, et al: IL-33 drives airway hyper-responsiveness through IL-13-mediated mast cell: Airway smooth muscle crosstalk. Allergy 70: 556-567, 2015.

14. Ahmed A and Koma MK: Interleukin-33 triggers B1 cell expansion and its release of monocyte/macrophage chemoattractants and growth factors. Scand J Immunol 82: 118-124, 2015.

15. Iijima K, Kobayashi T, Hara K, Kephart GM, Ziegler SF, McKenzie AN and Kita H: IL-33 and thymic stromal lymphopoietin mediate immune pathology in response to chronic airborne allergen exposure. J Immunol 193: 1549-1559, 2014.

16. Gordon ED, Simpson LJ, Rios CL, Ringel L, Lachowicz-Scroggins ME, Peters MC, Wesolowska-Andersen A, Gonzalez JR, MacLeod HJ, Christian LS, et al: Alternative splicing of interleukin-33 and type 2 inflammation in asthma. Proc Natl Acad Sci USA 113: 8765-8770, 2016. 
17. Hristova M, Habibovic A, Veith C, Janssen-Heininger YMW Dixon AE, Geiszt M and van der Vliet A: Airway epithelial dual oxidase 1 mediates allergen-induced IL-33 secretion and activation of type 2 immune responses. J Allergy Clin Immunol 137: 1545-1556, 2016.

18. Préfontaine D, Nadigel J, Chouiali F, Audusseau S, Semlali A, Chakir J, Martin JG and Hamid Q: Increased IL-33 expression by epithelial cells in bronchial asthma. J Allergy Clin Immunol 125 752-754, 2010

19. Guo Z, Wu J, Zhao J, Liu F, Chen Y, Bi L, Liu S and Dong L: IL-33 promotes airway remodeling and is a marker of asthma disease severity. J Asthma 51: 863-869, 2014.

20. Savenije OE, Mahachie John JM, Granell R, Kerkhof M, Dijk FN, de Jongste JC, Smit HA, Brunekreef B, Postma DS Van Steen K, et al: Association of IL33-IL-1 receptor-like 1 (IL1RL1) pathway polymorphisms with wheezing phenotypes and asthma in childhood. J Allergy Clin Immunol 134: 170-177, 2014.

21. Keenan CR, Salem S, Fietz ER, Gualano RC and Stewart AG: Glucocorticoid-resistant asthma and novel anti-inflammatory drugs. Drug Discov Today 17: 1031-1038, 2012.

22. He XF, Pan WD, Yao YL and Zhang HM: Recent highlights of Chinese herbs in treatment of allergic disease: Acting via mitogen-activated protein kinase signal pathway. Chin J Integr Med 23: 570-573, 2017.

23. Shao YY, Zhou YM, Hu M, Li JZ, Chen CJ, Wang YJ, Shi XY, Wang WJ and Zhang TT: The anti-allergic rhinitis effect of traditional Chinese medicine of shenqi by regulating mast cell degranulation and Th1/Th2 cytokine balance. Molecules 22: E504, 2017.

24. Li YQ, Wang JY, Qian ZQ, Li YL, Li WN, Gao Y and Yang DL: Osthole inhibits intimal hyperplasia by regulating the NF- $\kappa \mathrm{B}$ and TGF- $\beta 1 / \mathrm{Smad} 2$ signalling pathways in the rat carotid artery after balloon injury. Eur J Pharmacol 811: 232-239, 2017.

25. Gao Z, Wen Q, Xia Y, Yang J, Gao P, Zhang N, Li H and Zou S: Osthole augments therapeutic efficiency of neural stem cells-based therapy in experimental autoimmune encephalomyelitis. J Pharmacol Sci 124: 54-65, 2014.

26. Kordulewska NK, Kostyra E, Cieślińska A, Fiedorowicz E and Jarmołowska B: Cytokine production by PBMC and serum from allergic and non-allergic subjects following in vitro histamine stimulation to test fexofenadine and osthole anti-allergic properties. Eur J Pharmacol 791: 763-772, 2016.

27. Chen X, Pi R, Zou Y, Liu M, Ma X, Jiang Y, Mao X and $\mathrm{Hu} \mathrm{X}$ : Attenuation of experimental autoimmune encephalomyelitis in C57 BL/6 mice by osthole, a natural coumarin. Eur J Pharmacol 629: 40-46, 2010.

28. Hua KF, Yang SM, Kao TY, Chang JM, Chen HL, Tsai YJ, Chen A, Yang SS, Chao LK and Ka SM: Osthole mitigates progressive $\operatorname{Ig} \mathrm{A}$ nephropathy by inhibiting reactive oxygen species generation and NF- $\mathrm{B} / \mathrm{NLRP} 3$ pathway. PLoS One 8: e77794, 2013.

29. Matsuda H,Tomohiro N, Ido Y and Kubo M: Anti-allergic effects of cnidii monnieri fructus (dried fruits of Cnidium monnieri) and its major component, osthol. Biol Pharm Bull 25: 809-812, 2002.

30. Chiu PR, Lee WT, Chu YT, Lee MS, Jong YJ and Hung CH: Effect of the Chinese herb extract osthol on IL-4 induced eotaxin expression in BEAS-2B cells. Pediatr Neonatol 49: 135-140, 2008.

31. Sun J, Wu J, Xu C, Luo Q, Li B and Dong J: Paeoniflorin attenuates allergic inflammation in asthmatic mice. Int Immunopharmacol 24: 88-94. 2015.

32. Glaab T, Daser A, Braun A, Neuhaus-Steinmetz U, Fabel H, Alarie Y and Renz H: Tidal midexpiratory flow as a measure of airway hyperresponsiveness in allergic mice. Am J Physiol Lung Cell Mol Physiol 280: L565-L573, 2001.

33. Milstrey A, Wieskoetter B, Hinze D, Grueneweller N, Stange R, Pap T, Raschke M and Garcia P: Dose-dependent effect of parathyroid hormone on fracture healing and bone formation in mice. J Surg Res 220: 327-335, 2017.

34. Qiao Y, Song L, Zhu C, Wang Q, Guo T, Yan Y and LI Q: Dataset on preparation of the phosphorylated counterparts of a momordica charantia protein for studying antifungal activities against susceptible dose-dependent C. albicans to antimycotics. Data Brief 15: 370-375, 2017

35. Arancio AL, Cole KD, Dominguez AR, Cohenour ER, Kadie J, Maloney WC, Cilliers C and Schuh SM: Bisphenol A, Bisphenol AF, Di-N-Butyl phthalate, and 17 $\beta$-estradiol have shared and unique dose-dependent effects on early embryo cleavage divisions and development in Xenopus laevis. Reprod Toxicol 84: 65-74, 2019.
36. Schmittgen TD and Livak KJ: Analyzing real-time PCR data by the comparative C(T) method. Nat Protoc 3: 1101-1108, 2008

37. Virchow JC, Backer V, Kuna P, Prieto L, Nolte H, Villesen HH, Ljørring C, Riis B and de Blay F: Efficacy of a house dust mite sublingual allergen immunotherapy tablet in adults with allergic asthma: A randomized clinical trial. JAMA 315: 1715-1725, 2016.

38. Qian J, Ma X, Xun Y and Pan L: Protective effect of forsythiaside A on OVA-induced asthma in mice. Eur J Pharmacol 812: 250-255, 2017.

39. Wijerathne CUB, Seo CS, Song JW, Park HS, Moon OS, Won YS, Kwon HJ and Son HY: Isoimperatorin attenuates airway inflammation and mucus hypersecretion in an ovalbumin-induced murine model of asthma. Int Immunopharmacol 49: 67-76, 2017.

40. Grotenboer NS, Ketelaar ME, Koppelman GH and Nawijn MC: Decoding asthma: Translating genetic variation in IL33 and IL1RL1 into disease pathophysiology. J Allergy Clin Immunol 131: 856-865, 2013.

41. Pichery M, Mirey E, Mercier P, Lefrancais E, Dujardin A, Ortega $\mathrm{N}$ and Girard JP: Endogenous IL-33 is highly expressed in mouse epithelial barrier tissues, lymphoid organs, brain, embryos, and inflamed tissues: In situ analysis using a novel Il-33-LacZ gene trap reporter strain. J Immunol 188: 3488-3495, 2012.

42. Schmitz J, Owyang A, Oldham E, Song Y, Murphy E, McClanahan TK, Zurawski G, Moshrefi M, Qin J, Li X, et al: IL-33, an interleukin-1-like cytokine that signals via the IL-1 receptor-related protein ST2 and induces T helper type 2-associated cytokines. Immunity 23: 479-490, 2005.

43. Liew FY, Pitman NI and McInnes IB: Disease-associated functions of IL-33: The new kid in the IL-1 family. Nat Rev Immunol 10: 103-110, 2010.

44. Griesenauer B and Paczesny S: The ST2/IL-33 axis in immune cells during inflammatory diseases. Front Immunol 8: 475, 2017.

45. Bønnelykke K, Sleiman P, Nielsen K, Kreiner-Møller E, Mercader JM, Belgrave D, den Dekker HT, Husby A, Sevelsted A, Faura-Tellez G, et al: A genome-wide association study identifies CDHR3 as a susceptibility locus for early childhood asthma with severe exacerbations. Nat Genet 46: 51-55, 2014.

46. Wan Y, Shrine NR, Soler Artigas M, Wain LV, Blakey JD, Moffatt MF, Bush A, Chung KF, Cookson WO, Strachan DP, et al: Genome-wide association study to identify genetic determinants of severe asthma. Thorax 67: 762-768, 2012.

47. Morita H, Arae K, Unno H, Miyauchi K, Toyama S, Nambu A, Oboki K, Ohno T, Motomura K, Matsuda A, et al: An interleukin-33-mast cell-interleukin-2 axis suppresses papain-induced allergic inflammation by promoting regulatory $\mathrm{T}$ cell numbers. Immunity 43: 175-186, 2015.

48. Martin NT and Martin MU: Interleukin 33 is a guardian of barriers and a local alarmin. Nat Immunol 17: 122-131, 2016.

49. Chackerian AA, Oldham ER, Murphy EE, Schmitz J, Pflanz S and Kastelein RA: IL-1 receptor accessory protein and ST2 comprise the IL-33 receptor complex. J Immunol 179: 2551-2555, 2007.

50. Liew FY, Girard JP and Turnquist HR: Interleukin-33 in health and disease. Nat Rev Immunol 16: 676-689, 2016.

51. Drake LY and Kita H: IL-33: Biological properties, functions, and roles in airway disease. Immunol Rev 278: 173-184, 2017.

52. Jackson DJ, Makrinioti H, Rana BM, Shamji BWH, Trujillo-Torralbo MB, Footitt J, Del-Rosario J, Telcian AG, Nikonova A, Zhu J, et al: IL-33-dependent type 2 inflammation during rhinovirus-induced asthma exacerbations in vivo. Am J Respir Crit Care Med 190: 1373-1382, 2014.

53. Rank MA, Kobayashi T, Kozaki H, Bartemes KR, Squillace DL and Kita H: IL-33-activated dendritic cells induce an atypical TH2-type responses. J Allergy Clin Immunol 123: 1047-1054, 2009.

54. Kurowska-Stolarska M, Stolarski B, Kewin P, Murphy G, Corrigan CJ, Ying S, Pitman N, Mirchandani A, Rana B, van Rooijen N, et al: IL-33 amplifies the polarization of alternatively activated macrophages that contribute to airway inflammation. J Immunol 183: 6469-6477, 2009.

55. Millien VO, Lu W, Shaw J, Yuan X, Mak G, Roberts L, Song LZ, Knight JM, Creighton CJ, Luong A, et al: Cleavage of brinogen by proteinases elicits allergic responses through Toll-like receptor 4 . Science 341: 792-796, 2013

56. Yanagawa Y, Suzuki M, Matsumoto $M$ and Togashi $H$ : Prostaglandin E(2) enhances IL-33 production by dendritic cells. Immunol Lett 141: 55-60, 2011.

This work is licensed under a Creative Commons Attribution-NonCommercial-NoDerivatives 4.0 International (CC BY-NC-ND 4.0) License. 